\title{
Study of the association between the CAPSL-IL7R locus and type 1 diabetes
}

\author{
J. L. Santiago • B. Z. Alizadeh • A. Martínez • \\ L. Espino $\cdot$ H. de la Calle $\cdot$ M. Fernández-Arquero • \\ M. A. Figueredo • E. G. de la Concha • B. O. Roep • \\ B. P. C. Koeleman • E. Urcelay
}

Received: 29 April 2008 / Accepted: 16 May 2008 / Published online: 18 June 2008

(C) Springer-Verlag 2008

\begin{abstract}
Aims/hypothesis In the past few years, several genes outside the MHC region have been described as new susceptibility genetic factors to type 1 diabetes. An association between CAPSL-rs1445898 and type 1 diabetes was reported in a large white population and corroborated in a genome-wide analysis, which also found an association with $I L 7 R$, which is located adjacent to CAPSL. The aim of this study was to replicate the aforementioned associations in independent cohorts.

Methods We analysed two CAPSL (rs1010601 and rs1445898) and three $I L 7 R$ (rs6897932, rs987106 and rs3194051) polymorphisms. All these single nucleotide polymorphisms (SNPs) were genotyped using TaqMan minor groove binder chemistry in 301 unrelated Spanish type 1 diabetes patients and 646 healthy controls. Additionally, the associated CAPSL SNP rs1445898 was
\end{abstract}

J. L. Santiago $(\varangle) \cdot$ A. Martínez $\cdot$ L. Espino $\cdot$

M. Fernández-Arquero $\cdot$ M. A. Figueredo •

E. G. de la Concha $\cdot$ E. Urcelay

Department of Immunology, Hospital Clínico San Carlos,

Martín Lagos s/n,

28040 Madrid, Spain

e-mail: jlsantial@gmail.com

B. Z. Alizadeh • B. P. C. Koeleman

Department of Medical Genetics,

University Medical Center Utrecht,

Utrecht, the Netherlands

H. de la Calle

Endocrinology Department, Hospital Ramón y Cajal,

Madrid, Spain

B. O. Roep

Department of Immunohematology and Blood Transfusion, Leiden University Medical Center,

Leiden, the Netherlands genotyped in a Dutch cohort consisting of 429 type 1 diabetes patients and 720 healthy controls.

Results The homozygous mutant genotype of the CAPSL SNP rs 1445898 showed a trend towards a protective effect in the overall Spanish cohort (OR $[95 \% \mathrm{CI}] 0.70[0.44$ 1.09]; $p=0.09$ ) and in the Dutch cohort (OR [95\% CI] 0.74 [0.51-1.05]; $p=0.09$ ). Pooling of both cohorts was performed, yielding a statistically significant difference (Mantel-Haenszel OR $0.71 ; p=0.005$ ). This protective effect was significantly different in early-onset vs late-onset Spanish patients (OR [95\% CI] $0.26[0.10-0.65] ; p=0.001)$. Similarly, in the early-onset subgroup, the homozygous mutant genotype of the $I L 7 R$ SNP rs6897932 showed a similar protective effect (OR [95\% CI] 0.18 [0.02-0.94]; $p=0.02$ ). Conclusions/interpretation In summary, we describe an independent replication of the association between the CAPSL-IL7R locus and type 1 diabetes, especially for early-onset type 1 diabetes patients.

Keywords Age at onset · CAPSL · Diabetes · IL7R .

Polymorphisms

$\begin{array}{ll}\text { Abbreviations } \\ \text { CAPSL } & \text { calcyphosine-like } \\ \text { IL7R } & \text { IL-7 receptor } \\ \text { LD } & \text { linkage disequilibrium } \\ \text { SNP } & \text { single nucleotide polymorphism } \\ \text { WTCCC } & \text { Wellcome Trust Case Control Consortium }\end{array}$

\section{Introduction}

Type 1 diabetes is a complex disease resulting from the interaction between genetic and environmental factors that cause selective destruction of pancreatic beta cells. Despite 
a proven genetic component, the actual susceptibility genes are largely unknown. The genetic predisposition to type 1 diabetes is strongly associated with the MHC region; however, some loci outside the MHC have been considered to confer disease risk. Some of these have been validated, together with MHC class II alleles [1, 2], as susceptibility factors, namely the insulin gene [3, 4], the CTLA4 locus [5, 6] and the PTPN22 gene [7].

Smyth et al. [8] found an association between two new single nucleotide polymorphisms (SNPs) and type 1 diabetes in a genome-wide scan study of non-synonymous SNPs. The first new SNP was rs1445898 from the CAPSL gene (calcyphosine-like; also known as $Q 8 W W F 8$ ) on chromosome 5p13; the second was rs19907060 from the interferon induced with helicase $\mathrm{C}$ domain 1 (IFIH1) gene on chromosome $2 \mathrm{q} 24.3$. In that study, the authors reported that, leaving aside the MHC, the SNP most strongly associated with type 1 diabetes was located in the PTPN22 gene as described for the first time by Bottini et al. [7] and consistently replicated as a type 1 diabetes susceptibility locus in independent white populations. The second most strongly associated SNP was rs1445898 (Q75R) from CAPSL. In a later study of this group [9], the authors obtained evidence of disease association for three SNPs in the 5p13 region: the aforementioned SNP from CAPSL and two new SNPs located in $I L 7 R$ (also known as IL7Ralpha or $C D 127)$. These results are an extension of other genomewide analyses of 14,000 cases of seven common diseases that originally did not report an association between the 5 p13 region and diabetes [10]. Moreover, in a recent genome-wide association study in multiple sclerosis, an $I L 7 R$ polymorphism was identified to have a modest effect on the multiple sclerosis risk [11].

The function of calcyphosine-like (CAPSL) is still unknown, although it contains two calcium-binding motifs (EF-hands). These conserved domains are found in a superfamily of calcium sensors and calcium signal modulators. Furthermore, the CAPSL gene is in the same linkage disequilibrium (LD) block as the $I L 7 R$ gene. The IL-7 receptor (IL7R) is a heterodimer consisting of a specific alpha chain (encoded by $I L 7 R$ ) and a common gamma chain (encoded by $I L 2 R G$, also known as $C D 132$ and located in $\mathrm{Xq13.1)}$ that is shared by the receptors of several cytokines: IL-2, IL-4, IL-7, IL-9, IL-15 and IL-21. Whereas the gamma chain is produced by most haemopoietic cells, IL7R is almost exclusively produced by lymphoid lineage cells and is required for development and maintenance of the immune system [12]. In fact, mutations in $I L 7 R$ gene in human patients cause a severe combined immunodeficiency [13] in which the major deficiencies are in $\mathrm{T}$ cell development, whereas B and NK cells are relatively normal in numbers [14]. The expression of $I L 7 R$ is required by T cell progenitors, naive $\mathrm{T}$ cells and memory $\mathrm{T}$ cells. Its lack of expression correlates with altered immunity, making this gene a good candidate in association studies on autoimmune diseases.

The aim of the present study was to replicate the association between CAPSL and IL7R polymorphisms and type 1 diabetes in the Spanish population and to further replicate our findings in the Dutch population. Additionally, we sought to find out whether the putative CAPSL-IL7R effect occurs in paediatric cohorts only, as previously described [9], or whether it also extends to adult type 1 diabetes patients. We analysed the aforementioned SNP from CAPSL, which is located in the third exon (rs1445898), and another one (rs1010601) in the fourth intron. In the $I L 7 R$ gene, we selected three SNPs located in exon 6 (rs6897932, T244I), intron 6 (rs987106) and exon 8 (rs3194051, I356V).

\section{Methods}

Patients and controls We studied 301 white unrelated Spanish type 1 diabetes patients (150 women, $151 \mathrm{men})$ diagnosed according to the criteria of the American Diabetes Association (ADA) and 646 healthy controls, who were recruited from among blood donors in the Madrid area (therefore with an age ranging from 18 to 60 years).

The age at onset for the consecutively recruited type 1 diabetes patients ranged from 1 to 55 years old (mean: $17.3 \pm 10.0$ years; median age at onset 15 years). All patients were insulin-dependent at the time of the study. Informed consent was obtained from all participants included in the study, which was approved by the Ethics Committee of the Hospital Clínico San Carlos.

As cut-off age for stratified analysis, we decided to choose patients younger than 16 years, because 15 years is our median and in non-normalised variables that is the best statistical parameter to subdivide a cohort in order to maximise the statistical power.

In order to increase the statistical power, we included an additional Dutch cohort of 429 type 1 diabetes patients (median age at diagnosis 8.7 years, range 1-16 years) and 720 healthy unrelated controls. The diagnosis was made according to International Society of Paediatric and Adolescent Diabetes (ISPAD) criteria. All patients gave their informed consent and the Medical Ethics Committee of Leiden University Medical Centre approved this study.

Genotyping Patients and controls were genotyped for the CAPSL SNPs rs 1445898 and rs1010601 and for IL7R SNPs rs6891932, rs987106 and rs3194051. Genotyping was done using TaqMan assays in a 7900HT fast real-time PCR System (Applied Biosystems, Foster City, CA, USA). All assays (identification numbers: C_8811801_1, C_ 2025977_10, 
C $881185810, \mathrm{C} \quad 881180810$ and C 2561680510 , respectively) were performed as recommended by the manufacturer. Call rate success was over 97\%; genotyping was repeated in $10 \%$ of the samples and the results were consistent.

Statistical analysis Differences in allele and genotype frequencies were calculated by $\chi^{2}$ or Fisher's exact test when necessary. Associations were estimated by the OR with $95 \%$ CI. Statistical analysis used Epi Info v. 6.02 (CDC, Atlanta, GA, USA). No statistically significant deviations from Hardy-Weinberg equilibrium were observed for genotypes of these polymorphisms in our sample.

Maximum-likelihood haplotype frequencies were estimated by applying the expectation maximisation (EM) and partition ligation (PL) algorithms, both implemented by Haploview 4.0 software [15]. Linkage disequilibrium was calculated using the algorithm from Gabriel et al. implemented in Haploview 4.0 software [16].
The Mann-Whitney Test was performed by SPSS 13.0 software (SPSS, Chicago, IL, USA).

The Mantel-Haenszel method was performed by EpiDat 3.1 software (http://dxsp.sergas.es)

\section{Results}

The analysis in our overall Spanish cohort showed a trend towards a protective effect only for the homozygous mutant genotype of the CAPSL SNP rs1445898 (Table 1). To increase the statistical power, we included the analysis, in a Dutch cohort, of this polymorphism, which is the most strongly associated SNP in the Todd et al. study [9]. In agreement with the results obtained in our Spanish cohort, a trend towards a protective effect was also found here (Table 2). Meta-analysis was conducted using the MantelHaenszel method and yielded a significant association: OR (95\% CI) $0.71(0.56-0.90) ; p=0.005$.

Table 1 Allelic and genotypic frequencies from $I L 7 R$ and CAPSL polymorphisms in the Spanish cohort

\begin{tabular}{|c|c|c|c|c|}
\hline Parameter & Type 1 diabetes, $n(\%)$ & Controls, $n(\%)$ & OR $(95 \% \mathrm{CI})$ & $p$ value \\
\hline \multicolumn{5}{|c|}{$I L 7 R$ rs6897932 } \\
\hline $\mathrm{T}$ & $147(24.4)$ & $333(25.8)$ & \multirow[t]{2}{*}{$0.93(0.47-1.17)$} & \multirow[t]{3}{*}{0.53} \\
\hline $\mathrm{C}$ & $455(75.6)$ & $959(74.2)$ & & \\
\hline $\mathrm{CC}$ & $169(56.1)$ & $352(54.5)$ & 1.00 (reference) & \\
\hline $\mathrm{CT}$ & $117(38.9)$ & $255(39.5)$ & $0.96(0.71-1.28)$ & 0.76 \\
\hline $\mathrm{TT}$ & $15(5.0)$ & $39(6.0)$ & $0.80(0.41-1.55)$ & 0.48 \\
\hline \multicolumn{5}{|c|}{$I L 7 R$ rs987106 } \\
\hline $\mathrm{T}$ & $269(44.7)$ & $605(46.8)$ & \multirow[t]{2}{*}{$0.92(0.75-1.12)$} & \multirow[t]{3}{*}{0.38} \\
\hline A & $333(55.3)$ & $687(53.2)$ & & \\
\hline AA & 99 (32.9) & $193(29.9)$ & 1.00 (reference) & \\
\hline AT & $135(44.9)$ & $301(46.6)$ & $0.87(0.63-1.21)$ & 0.41 \\
\hline $\mathrm{TT}$ & $67(22.3)$ & $152(23.5)$ & $0.86(0.58-1.27)$ & 0.43 \\
\hline \multicolumn{5}{|c|}{$I L 7 R$ rs3194051 } \\
\hline $\mathrm{G}$ & $182(30.2)$ & $384(29.7)$ & \multirow[t]{2}{*}{$1.02(0.83-1.27)$} & \multirow[t]{3}{*}{0.82} \\
\hline A & $420(69.8)$ & $908(70.3)$ & & \\
\hline AA & $141(46.8)$ & $310(48.0)$ & 1.00 (reference) & \\
\hline $\mathrm{AG}$ & $138(45.9)$ & $288(44.6)$ & $1.05(0.78-1.41)$ & 0.72 \\
\hline GG & $22(7.3)$ & $48(7.4)$ & $1.01(056-1.79)$ & 0.98 \\
\hline \multicolumn{5}{|c|}{ CAPSL rs1010601 } \\
\hline $\mathrm{C}$ & $265(44.0)$ & $528(40.9)$ & \multirow[t]{2}{*}{$1.14(0.93-1.39)$} & \multirow[t]{3}{*}{0.20} \\
\hline $\mathrm{T}$ & $337(56.0)$ & $764(59.1)$ & & \\
\hline $\mathrm{TT}$ & $90(29.9)$ & $218(33.7)$ & 1.00 (reference) & \\
\hline $\mathrm{TC}$ & $157(52.2)$ & $328(50.7)$ & $1.16(0.84-1.60)$ & 0.35 \\
\hline $\mathrm{CC}$ & 54 (17.9) & $100(15.5)$ & $1.31(0.85-2.02)$ & 0.20 \\
\hline \multicolumn{5}{|c|}{ CAPSL rs1445898 } \\
\hline $\mathrm{T}$ & $255(42.4)$ & $588(45.5)$ & \multirow[t]{2}{*}{$0.88(0.72-1.07)$} & \multirow[t]{3}{*}{0.20} \\
\hline $\mathrm{C}$ & 347 (57.6) & $704(54.5)$ & & \\
\hline $\mathrm{CC}$ & $89(29.6)$ & $190(29.4)$ & 1.00 (reference) & \\
\hline $\mathrm{CT}$ & $169(56.1)$ & $324(50.2)$ & $1.11(0.80-1.54)$ & 0.50 \\
\hline $\mathrm{TT}$ & $43(14.3)$ & $132(20.4)$ & $0.70(0.44-1.09)$ & 0.09 \\
\hline
\end{tabular}

Type 1 diabetes $n=301$; controls $n=646$ 
Table 2 Allelic and genotypic frequencies of CAPSL SNP rs1445898 in a Dutch cohort

\begin{tabular}{lccc}
\hline CAPSL rs1445898 & Type 1 diabetes, $n(\%)$ & Controls, $n(\%)$ & OR (95\% CI) \\
\hline T & $339(40.5)$ & $626(43.8)$ & $0.87(0.73-1.04)$ \\
C & $499(59.5)$ & $802(56.2)$ & 0.12 \\
CC & $149(35.5)$ & $236(33.1)$ & $1.00($ reference $)$ \\
CT & $201(48.0)$ & $330(46.2)$ & $0.96(0.73-1.26)$ \\
TT & $69(16.5)$ & $148(20.7)$ & $0.74(0.51-1.05)$ \\
\hline
\end{tabular}

No significant differences were found in genotypes of any of the five markers in the groups when stratified by sex (data not shown). The classical stratification by $H L A-D R B 1$ susceptibility alleles (antigen specificity DR3 and DR4) evidenced no significant differences in genotype frequencies of any of the five SNPs between carriers and non-carriers of MHC class II susceptibility alleles (data not shown).

However, in the case of age at disease onset in our Spanish cohort, the protective effect of the minor allele and the homozygous mutant genotype of the CAPSL SNP rs1445898 was statistically significant in young-onset patients compared with the group older than 15 years at onset (Table 3). Interestingly, a similar protective effect was seen in young patients carrying either the T allele or TT genotype in the IL7R SNP rs6897932 marker (Table 3). A significant difference was found between young patients and controls for the TT genotype in both markers (IL7R SNP rs6897932: OR 0.22 [0.02-0.88]; $p=0.02 ; C A P S L$

Table 3 Distribution of $I L 7 R$ and CAPSL polymorphisms in Spanish patients stratified by age at onset of type 1 diabetes

\begin{tabular}{|c|c|c|c|c|}
\hline Parameter & Age $\leq 15$ years, $n(\%)$ & Age $>15$ years, $n(\%)$ & OR $(95 \% \mathrm{CI})$ & $p$ value \\
\hline \multicolumn{5}{|c|}{$I L 7 R$ rs6897932 } \\
\hline $\mathrm{T}$ & $54(20.1)$ & $75(28.0)$ & $0.65(0.43-0.99)$ & 0.03 \\
\hline $\mathrm{C}$ & $214(79.9)$ & $193(72.0)$ & & \\
\hline $\mathrm{CC}$ & $82(61.2)$ & $68(50.7)$ & 1.00 (reference) & \\
\hline $\mathrm{CT}$ & $50(37.3)$ & $57(42.5)$ & $0.73(0.43-1.23)$ & 0.21 \\
\hline $\mathrm{TT}$ & $2(1.5)$ & $9(6.7)$ & $0.18(0.02-0.94)$ & 0.02 \\
\hline \multicolumn{5}{|c|}{$I L 7 R$ rs987106 } \\
\hline $\mathrm{T}$ & $123(45.9)$ & $115(42.9)$ & $1.13(0.79-1.61)$ & 0.49 \\
\hline A & $145(54.1)$ & $153(57.1)$ & & \\
\hline AA & $42(31.3)$ & $47(35.1)$ & 1.00 (reference) & \\
\hline AT & $61(45.5)$ & $59(44.0)$ & $1.16(0.64-2.08)$ & 0.60 \\
\hline $\mathrm{TT}$ & $31(23.1)$ & $28(20.9)$ & $1.24(0.61-2.53)$ & 0.52 \\
\hline \multicolumn{5}{|c|}{$I L 7 R$ rs 3194051} \\
\hline $\mathrm{G}$ & $91(34.0)$ & $74(27.6)$ & $1.35(0.92-1.98)$ & 0.11 \\
\hline A & $177(66.0)$ & $194(72.4)$ & & \\
\hline AA & $54(40.3)$ & $69(51.5)$ & 1.00 (reference) & \\
\hline $\mathrm{AG}$ & $69(51.5)$ & $56(41.8)$ & $1.57(0.92-2.68)$ & 0.08 \\
\hline GG & $11(8.2)$ & $9(6.7)$ & $1.56(0.55-4.46)$ & 0.36 \\
\hline \multicolumn{5}{|c|}{ CAPSL rs 1010601} \\
\hline $\mathrm{C}$ & $128(47.8)$ & $110(41.0)$ & $1.31(0.92-1.88)$ & 0.12 \\
\hline $\mathrm{T}$ & $140(52.2)$ & $158(59.0)$ & & \\
\hline TT & $34(25.4)$ & $42(31.3)$ & 1.00 (reference) & \\
\hline $\mathrm{TC}$ & $72(53.7)$ & $74(55.2)$ & $1.20(0.66-2.18)$ & 0.52 \\
\hline $\mathrm{CC}$ & $28(20.9)$ & $18(13.4)$ & $1.92(0.86-4.34)$ & 0.08 \\
\hline \multicolumn{5}{|c|}{ CAPSL rs 1445898} \\
\hline $\mathrm{T}$ & $96(35.8)$ & $128(47.8)$ & $0.61(0.43-0.88)$ & 0.005 \\
\hline $\mathrm{C}$ & $172(64.2)$ & $140(52.2)$ & & \\
\hline fCC & $48(35.8)$ & $32(23.9)$ & 1.00 (reference) & \\
\hline $\mathrm{CT}$ & $76(56.7)$ & $76(56.7)$ & $0.67(0.37-1.20)$ & 0.15 \\
\hline TT & $10(7.5)$ & $26(19.4)$ & $0.26(0.10-0.65)$ & 0.001 \\
\hline
\end{tabular}

Age $\leq 15$ years $n=134$; age $>15$ years $n=134$

The total number of patients was 268 because age at disease onset could not be ascertained for 33 patients 
Fig. $1 D^{\prime}$ values (a) and $r^{2}$ values (b) calculated in the Spanish control cohort (Haploview 4.0 software [15]) a
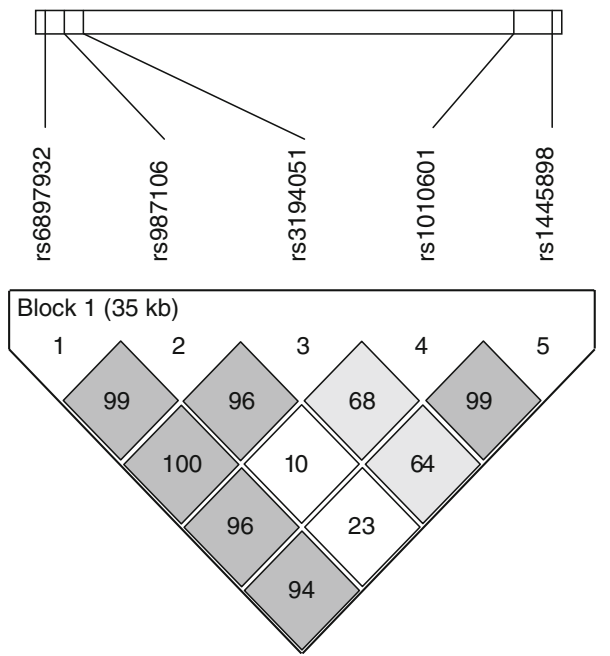

b
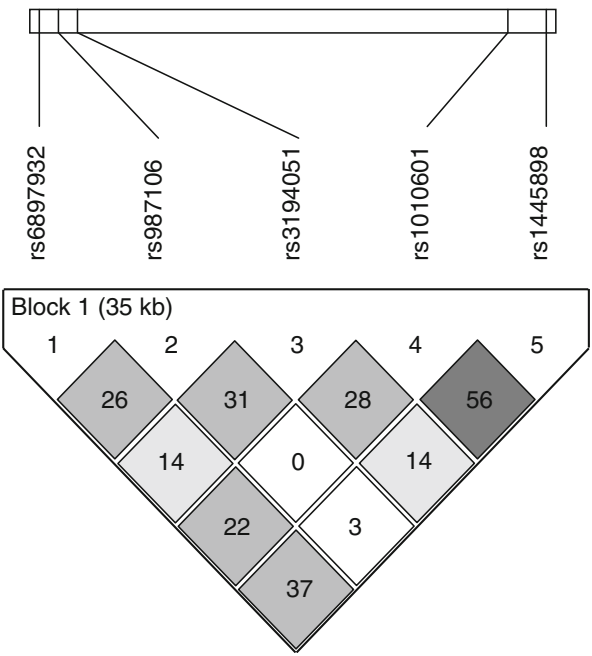

SNP rs1445898: OR 0.30 [0.14-0.64]; $p=0.0006)$, but no difference was found between patients older than 15 years and controls for the same markers (IL7R SNP rs6897932: OR 1.19 [0.51-2.71]; $p=0.65 ; C A P S L$ SNP rs1445898: OR 1.17 [0.64-2.13]; $p=0.59)$. Performing the analysis of age as a continuous variable, we found a trend for association of CAPSL SNP rs1445898 using the non-parametric MannWhitney test: asymptotic significance (two-tailed) $=0.072$. As the Dutch patients were all paediatric, we merged these samples with the paediatric Spanish samples, and found a strongly protective effect (Mantel-Haenszel: OR 0.62 [0.46-0.91]; $p=0.0007$ ).

Finally, high LD in the control cohort was found in the $I L 7 R-C A P S L$ region, but the five markers tested were not genetically equivalent (Fig. 1). The study of inferred haplotypes conformed by the five polymorphisms showed no differences between non-stratified cases and controls. After stratification by age at disease onset, haplotypes did not add information to that provided by the isolated polymorphisms (data not shown).

\section{Discussion}

Whole-genome association screenings in type 1 diabetes have confirmed the importance of the HLA region and uncovered non-HLA loci that may harbour susceptibility genes. We have studied one of these new regions on chromosome 5 p13 [8, 9]. The same SNP from CAPSL (rs1445898) that was already described showed a trend towards association in our Spanish and Dutch cohorts, supporting the role of this locus in type 1 diabetes risk (Tables 1 and 2). The effect is only evident in the homozygous mutant genotype, probably due either to compromised power of our study or to the weak protective effect in the general population. When the cohorts were pooled, the $p$ value was statistically significant (MantelHaenszel: $p=0.005$ ).

After stratification for age at onset, the protective effect concentrated in the young group was not only statistically significant for the allelic frequencies or for the homozygous mutant genotype (Table 3), but also for the carriers of the $\mathrm{T}$ allele (OR 0.56 [0.32-0.99]; $p=0.03$ ). The previously reported association was found in paediatric patients (all under 17 years at diagnosis) [9]; for this reason we decided to repeat the analysis in our Spanish paediatric cohort using the median as the cut-off age (Table 3). Nevertheless, when we subdivided patients in the same way as Todd et al. (younger than 17 years), we still found significant differences between both groups of patients (TT genotype of CAPSL SNP rs1445898 in type 1 diabetes $<17$ years vs type 1 diabetes $>16$ years: $13 / 49$ young-onset patients vs $23 / 31$ patients older than 16 years at onset, OR $0.36[0.15-$ 0.87]; $p=0.01)$. These findings suggest that type 1 diabetes patients with an early age of onset have a different disease mechanism than patients with adult-onset type 1 diabetes. Further study is needed to validate this effect and to elucidate the exact nature of this putative difference.

As shown in Table 3, the frequency of genotype TT of $I L 7 R$ SNP rs6897932 was significantly reduced in the young patients. This protective effect in the same group of patients provides additional confirmation of the importance of that LD block, where both genes map.

Two recent genome-wide association studies have reported that several non-MHC regions are associated with type 1 diabetes. In the first genome-wide association scan [10], carried out by the Wellcome Trust Case Control Consortium (WTCCC), some evidence was obtained for the association of this region using 2000 British patients. The WTCCC study did not include the most strongly associated SNP (CAPSL rs1445898), while the other associated SNP 
(IL7R rs6897932) showed a protective effect, although it did not exceed the threshold of significance for that study (TT genotype type 1 diabetes vs controls: $120 / 1100$ patients vs 235/1544 controls: OR 0.72 [0.56-0.91]; $p=0.005$ ). Todd et al. [9] in a follow-up genome-wide analysis also obtained evidence of disease association for rs1445898 in $C A P S L$ and rs6897932 in $I L 7 R$. They suggested that either geographical variability in SNP frequencies or population structure could increase the false-positive rate in casecontrol association studies. However, our data seem to indicate that those could be validated associations, because our replications in two independent populations mirror those previously found.

We consider that the CAPSL-IL7R locus is a protective region, but we cannot elucidate whether the protective gene is CAPSL, IL7R or both. The functional effect of these polymorphisms must be established. Although the function of CAPSL is not yet known, IL7R is a specific IL-7 receptor and this cytokine is essential for thymic maturation and for the homeostatic proliferation of lymphocytes [17]. Consequently, altered activity of IL-7 could influence the survival and the diversity of the $\mathrm{T}$ cell repertory, including the development of autoimmune T cells. NOD mice model studies have demonstrated that dendritic cells with inhibited expression of CD40, CD80 and CD86 confer a delay in diabetes incidence [18]. This process is carried out in part through the enhancement of $\mathrm{CD} 4^{+} \mathrm{CD} 25^{+} \mathrm{T}$ regulatory cells. Both in vivo and in vitro experiments have demonstrated that IL7R is expressed at significantly higher levels on T regulatory cells than on $\mathrm{CD} 4^{+} \mathrm{CD} 25^{-}$cells [19]. Although these observations could explain the protective effect of the IL-7 and IL7R pathway, additional functional studies will be necessary to understand the underlying immunoregulatory mechanism.

In conclusion, we confirmed the previously reported association between the CAPSL-IL7R locus and type 1 diabetes. Moreover, we have also described for the first time that this locus confers a protective effect specifically in type 1 diabetes patients with an early age at disease onset. This study warrants replication.

Acknowledgements We thank C. Martínez for expert technical assistance, A. Martínez and J. L. Santiago are recipients of FIS contracts (CP04/00175 and CM05/00216, respectively). E. Urcelay works for the "Fundación para la Investigación Biomédica- Hospital Clínico San Carlos".

The study was also supported by grants from the Dutch Diabetes Research Foundation (97.137), the Netherlands Organization for Health Research and Development (ZonMW) and the Juvenile Diabetes Research Foundation International (JDRF) (2001.10.004).

Duality of interest The authors declare that there is no duality of interest associated with this manuscript.

\section{References}

1. Concannon P, Erlich HA, Julier C et al (2005) Type 1 diabetes: evidence for susceptibility loci from four genomewide linkage scans in 1,435 multiplex families. Diabetes 54: 2995-3001

2. Pociot F, McDermott MF (2002) Genetics of type 1 diabetes mellitus. Genes Immun 3:235-249

3. Barratt BJ, Payne F, Lowe CE et al (2004) Remapping the insulin gene/IDDM2 locus in type 1 diabetes. Diabetes 53:1884-1889

4. Bell GI, Horita S, Karam JH (1984) A polymorphic locus near the human insulin gene is associated with insulin-dependent diabetes mellitus. Diabetes 33:176-183

5. Nistico L, Buzzetti R, Pritchard LE et al (1996) The CTLA-4 gene region of chromosome $2 \mathrm{q} 33$ is linked to, and associated with, type 1 diabetes. Belgian Diabetes Registry. Hum Mol Genet 5:1075-1080

6. Ueda H, Howson JM, Esposito L et al (2003) Association of the $\mathrm{T}$ cell regulatory gene CTLA4 with susceptibility to autoimmune disease. Nature 423:506-511

7. Bottini N, Musumeci L, Alonso A et al (2004) A functional variant of lymphoid tyrosine phosphatase is associated with type I diabetes. Nat Genet 36:337-338

8. Smyth DJ, Cooper JD, Bailey R et al (2006) A genome-wide association study of nonsynonymous SNPs identifies a type 1 diabetes locus in the interferon-induced helicase (IFIH1) region. Nat Genet 38:617-619

9. Todd JA, Walker NM, Cooper JD et al (2007) Robust associations of four new chromosome regions from genome-wide analyses of type 1 diabetes. Nat Genet 39:857-864

10. Wellcome Trust Case Control Consortium (2007) Genome-wide association study of 14,000 cases of seven common diseases and 3,000 shared controls. Nature 447:661-678

11. Hafler DA, Compston A, Sawcer S et al (2007) Risk alleles for multiple sclerosis identified by a genomewide study. N Engl J Med 357:851-862

12. Kondo M, Takeshita T, Higuchi M et al (1994) Functional participation of the IL-2 receptor gamma chain in IL-7 receptor complexes. Science 263:1453-1454

13. Roifman CM, Zhang J, Chitayat D, Sharfe N (2000) A partial deficiency of interleukin-7R alpha is sufficient to abrogate $T$ cell development and cause severe combined immunodeficiency. Blood 96:2803-2807

14. Giliani S, Mori L, de Saint Basile G et al (2005) Interleukin-7 receptor alpha (IL-7Ralpha) deficiency: cellular and molecular bases. Analysis of clinical, immunological, and molecular features in 16 novel patients. Immunol Rev 203:110-126

15. Barrett JC, Fry B, Maller J, Daly MJ (2005) Haploview: analysis and visualization of $\mathrm{LD}$ and haplotype maps. Bioinformatics 21:263-265

16. Gabriel SB, Schaffner SF, Nguyen H et al (2002) The structure of haplotype blocks in the human genome. Science 296:2225-2229

17. Watanabe M, Ueno $Y$, Yajima $T$ et al (1995) Interleukin 7 is produced by human intestinal epithelial cells and regulates the proliferation of intestinal mucosal lymphocytes. J Clin Invest 95:2945-2953

18. Machen J, Harnaha J, Lakomy R, Styche A, Trucco M, Giannoukakis N (2004) Antisense oligonucleotides down-regulating costimulation confer diabetes-preventive properties to nonobese diabetic mouse dendritic cells. J Immunol 173:4331-4341

19. Harnaha J, Machen J, Wright $M$ et al (2006) Interleukin-7 is a survival factor for CD4+ CD25+ T cells and is expressed by diabetes-suppressive dendritic cells. Diabetes 55:158-170 